The final publication is available at Springer via http://dx.doi.org/10.1007/s10796-017-9789-4 


\title{
Institutional vs. Non-Institutional Use of Social Media during Emergency Response: A Case of Twitter in 2014 Australian Bush Fire
}

Babak Abedin (contact author),

Faculty of Engineering \& IT, University of Technology Sydney, 15 Broadway, Ultimo, 2007 NSW, Australia

Babak.Abedin@uts.edu.au; Phone:+61295141834

Abdul Babar

Sydney Business School, University of Sydney, Camperdown, 2006 NSW, Australia,

Abdul.Babar@sydney.edu.au; Phone: +61291141342

\begin{abstract}
Social media plays a significant role in rapid propagation of information when disasters occur. Among the four phases of disaster management life cycle: prevention, preparedness, response, and recovery, this paper focuses on the use of social media during the response phase. It empirically examines the use of microblogging platforms by Emergency Response Organisations (EROs) during extreme natural events, and distinguishes the use of Twitter by EROs from digital volunteers during a fire hazard occurred in Australia state of Victoria in early February 2014. We analysed 7,982 tweets on this event. While traditionally theories such as World System Theory and Institutional Theory focus on the role of powerful institutional information outlets, we found that platforms like Twitter challenge such notion by sharing the power between large institutional (e.g. EROs) and smaller non-institutional players (e.g. digital volunteers) in the dissemination of disaster information. Our results highlight that both large EROs and individual digital volunteers proactively used Twitter to disseminate and distribute fire related information. We also found that the contents of tweets were more informative than directive, and that while the total number of messages posted by top EROs were more than the non-institutional ones, whereas non-institutions exceeded on tweets and sharing of the tweets.
\end{abstract}

\section{Keywords}

Social media, Twitter, Australian case study, emergency response organisations, community resilience 


\section{INTRODUCTION}

Disasters are complex in nature, which may have disproportionate effects at varying speed. Information exchange is pivotal during the disaster management processes (i.e., prevention, preparedness, response, and recovery), particularly in the response phase, as the dynamic and complex nature of the extreme natural disasters increases the rate of communication between stakeholders (Abedin et al. 2014).

Social media sites have been increasingly used by government organizations for communication with citizens to enhance a community's capacity in the face of disasters (Polet et al. 2017). Recent studies indicate that social media platforms support various activities within the disaster management cycle. For example, Houston et al. (2015) propose a functional framework for guiding the use of social media for disaster related research. This framework provides guidance on the use of various types of social media tools that can be used to perform a variety of disaster related activities ranging from preparing and receiving disaster preparedness information to disaster presentation initiatives and warnings as well as predicting disasters. Twitter, in particular, has gone beyond simply a social networking tool to a strategic communication tool during disastrous events all over the world (Lee et al. 2015). It has been used in pushing Toyota recalls in 2010 (Stieglitz and Krüger 2011) and Seattle Café shooting in 2012 (McNerthney 2012). It also has been used to manage crisis such as Victorian floods (Ehnis and Bunker 2012), Earthquake of Morgan Hill CA (Crooks et al. 2013), Haiti earthquake (Yates and Paquette 2011), and Kenya's Westgate mall terror attack (Simon et al. 2014).

Research shows that institutional actors actively use social media platforms like Twitter and Facebook in their formal interactions with public through sending them warnings and specific advice or directions (Whitelaw and Henson 2014; Erfani et al. 2016). Previous studies on institutional theory suggest that institutions normally actively conform to the demands of social media and may develop indirect forms of maintaining control over usergenerated content and the behaviours of social media users (Falls 2013). According to Scott (1995:53), "institutions are social structures that have attained a high degree of resilience. [They] are composed of culturalcognitive, normative, and regulative elements that, together with associated activities and resources, provide stability and meaning to social life". This perspective is in line with World System Theory (WST) (Wallerstein 1974), as the theory too promotes the notion that information and news typically flows from powerful outlets to minor peripheral information outlets (Barnett 2001). WST theory has roots in the practice of journalism, however it has been applied to various disciplines including information systems and management (Golan \& Himelboim 2016), and is a great tool to explain a more traditional perspective on information flow in any system. This theory suggests that all players in an information/news dissemination network act within a particular system, and that major institutional actors dominate the flow of information/news to non-institutional or minor institutional stakeholders.

Given the widespread use of social media by public, government, and non-government organizations, often the traditional perspectives such as those of WST on information dissemination between various stakeholders in a network (e.g. a disaster events) have been challenged. For example, in a recent study by Golan and Himelboim (2016) it was found that the structure of the information flow on Twitter displays a hierarchical core-periphery structure; however non-institutional stakeholders such as individual Twitter users conform less than institutional actors such as government organizations to that structure. The above scholars also discovered that while traditionally only a small number of formal institutions were supposed to deliver information to their audience, non-institutional actors assume the role of bridging news sources and audiences using social media tools and platforms.

While there are many studies in the literature on the use of social media during natural disasters, most of them have focused on institutional actors and how major organizations or government authorities use these tools to communicate with the people affected by disasters (Lerman 2010). However, non-government and local community members also play a significant role in information dissemination during disasters. For example, Bird et al. (2012) investigated the use of social media during the Queensland and Victoria floods in Australia, and found that community-initiated Facebook groups play a significant role in helping residents finding their friends and family, updating people with real time updates and providing information about the disaster as well as about government departments' activities, and also assisting travelers with information about road closure and necessary advices. Their research found that social media is not only a tool for information dissemination, but also a critical resource to tap into and utilize informal communications.

Thus, this paper challenges the predictions of WST and other similar perspectives on the flow of information between institutional and non-institutional actors in a given network or system. In particular, this paper aims to study the role of non-institutional and informal Twitter account holders in dissemination of disaster information. To address this need, we examine how the task of information dissemination was shared between 
Australian Emergency Response Organisations (EROs) and smaller non-institutional players such as digital volunteers and active individual Twitter account holders during Victorian fire hazard in 2014.

\section{SOCIAL MEDIA IN DISASTER MANAGEMENT}

Unlike traditional information and communication technologies, Social Media platforms such as Facebook, Twitter, Wikis, and SharePoint enable open and online exchange of information via conversation, interaction and exchange of user generated contents (Lee et al. 2015; Abedin \& Jafarzadeh, 2013). These platforms help people to establish connections and links with like-minded people and community members (Simon 2015). Social media users can share and discuss their opinions in real time through posts and links. Therefore, social media can be used to mobilize and organize communities in order to achieve various types of objectives, and update the communities with the most up-to-date information, which might not be available via traditional sources (Abedin 2016; Lerman 2010).

Social media platforms have increasingly been part of disaster response (Sarcevic 2012). These platforms which were previously used during disasters and emergencies by general public, are now being utilized by EROs, Governments, and non-Governmental organisations to manage disasters. During natural disasters social media provides access to relevant and timely information from both official and non-official sources which can help to facilitate a feeling of connectedness (Taylor 2012). This provides connectivity to loved ones, and brings comfort for the community as well as support and assistance to potentially distressed individuals and populations (Taylor 2012).

The utilization of social media for communications during disasters/emergencies was initiated by the public before emergency authorities start utilizing it. Apparently, the public wanted to find alternative ways (alternative to the unidirectional communication approach) to search and share updated information concerning the emergency (Sutton 2008). EROs now utilize social media to engage citizens in the emergency management by both disseminating information and accessing information from the public.

According to Latonero (2010), research on the use of social media during emergencies can be divided into two main categories: one that focuses on the ways in which EROs use social media to coordinate activities during their response to the event, and the other one that deals with the ways the public and victims share information on social media during emergencies. For examples, social media platforms were used to link the public with day-to-day, real-time information in preparation to the 2009H1N1 pandemic. A few minutes after the Alexandria, Virginia health department tweeted regarding availability and location of vaccines, people rushed to the vaccination sites (Merchant 2011). This presents that an integration of social media platforms for preparedness activities can facilitate an effective response from EROs as well as the citizens by using familiar platforms during an event (Merchant 2011). Since the 2010 earthquake in Haiti, EROs around the world have adopted social media as an important additional communication channel to communicate with the public (Bird 2012; Sarcevic 2012).

\section{THE STUDY EVENT}

Being the world's sixth largest country by area, Australia has got its share of natural disasters. It has a long and dreadful history of natural disasters such as bushfires, floods, severe storms, and landslides (Ahmad 2011). In recent years, Australian government agencies have shown a significant interest in the use of social media, particularly Twitter, for interaction with general public during emergency response (Whitelaw and Henson 2014; Alam and Walker 2011; Kavanaugha et al. 2011). The growth of Twitter users in Australia resembles the growth of Twitter users in many other countries including the United States (Whitelaw and Henson 2014).

Ehnis and Bunker (2012) found that Queensland Police Service (QPS) used microblogging services to interact with public during the 2011 Queensland flood disaster. Being proactive, QPS created an online community of followers before the flooding occurred. QPS conducted an extensive trial use of media technologies - Facebook, Twitter and YouTube, in which they wanted to establish a new channel for a two-way communication and collaboration between QPS and the public. These social media services were used to disseminate information and warning out to their following communities and general public. The "likes" and comments from the community indicated that the community did not only read information, but also seek interaction with the serving agencies with offer of help and assistance. But Ehnis and Bunker (2012) concluded it is still unclear that if the presence of government institutions on social media platform was effective in recent disasters.

In February 2014, Victoria confronted its worst bushfires since 2009 Black Saturday. A series of blazes, caused by the lightning strikes lit up the state near the end of January. The most serious bursts were in and around Melbourne in the first week of February, which destroyed three houses in an intense blaze at Warrandyte on the city's north eastern peripheral (SBS 2014). While the bushfire failed to affect power generation directly, around 12,000 Victorian homes suffered outages due to spikes in demand and damage to power lines. Fires particularly at Mickleham and Warrandyte moved fast with the wind change early in the afternoon, opening up new and wider 
fronts. More than 350 fire fighters and 60 trucks were deployed to battle the fire in Mickleham and Warrandyte, whereas a further 126 trucks fought the 3000-hectare fire in Gisborne and nearby Riddells Creek. According to the Country Fire Authority's (CFA) announcement the fires burning near Warrandyte, in Melbourne's outer North East, and near Gisborne, North West of the city were eased by 17 of Feb (SBS 2014). No one was killed or seriously injured during the Melbourne fire, but many animals were among the casualties of the 333,000-hectare fires 1 . In this event, the emergency authorities and the general public (people on the ground) heavily used Twitter to exchange information. Emergency management organizations used Twitter platform to provide up to minute information to the people whereas the individuals and social communities used Twitter to learn and forward that information by re-tweeting. In the 13 days of the fires, both emergency authorities and people on the ground posted around 7,981 tweets about these fires.

\section{METHODOLOGY AND DATA COLLECTION}

Analysing tweets, re-tweets, and mentions is a common practice in the majority of the studies on the use of Twitter (Mendoza et al. 2010; Simon et al. 2014; Oh et al. 2013; Crooks et al. 2013). For example Simon et al. (2014) investigated tweeting, re-tweeting and mentioning of government officials and security forces accounts during a terrorist attack in a shopping mall in Kenya. Such analysis helps to understand how Twitter has been used by various stakeholders such as general public, volunteers, media, emergency response organizations and so forth.

We used $\mathrm{R}$ with twitteR, tm, and sna packages which have also been previously used in the literature (Butts 2008; Díaz-Uriarte and Andrés 2006), for the collection, storage and analysis of Twitter data on Victoria fire. R, as an open source software environment for statistical computing and visualization, is used in conjunction with a variety of packages for improved, collection, analysis and storage of data in various areas of research such as epidemiological (Chongsuvivatwong 2008), bioinformatics (Heyde et al. 2014), environmental statistics, economics, medical and public health application.

- The R package 'twitteR' ${ }^{\prime 2}$ is an $\mathrm{R}$ based Twitter client which provides an interface to the Twitter web AP, for the collection and storage of Twitter data. It has been recognized as a simple and practical way for retrieving data from Twitter. It captures public tweets, trending topics and tweets containing a given hashtag, words, and user names. After collecting the desired tweets, we extracted their contents by using function twListToDF that puts everything in the data frame.

- $\quad \mathrm{R}$ package ' $\mathrm{tm}$ ' has also been used to perform text mining. The structure for managing documents in $\mathrm{tm}$ is called Corpus referring to a collection of text documents.

- The R package 'sna' is based on network theory for the purpose of analysing social networks consisting of nodes representing individual actors within the network.

Our first step in relation to data collection was to establish a secure and authentic connection with Twitter by performing handshake procedures, which includes using 'ROAuth' package with 'twitteR' package to setup credentials in the form of consumer key and secret. Once the connection was completed, the next step was to access data by using twitterR. The crawling and data collection occurred between 7 and 14 Feb 2014, which was the peak time period of the fire event, and we were able to extract 7,981 tweets created between 20 Jan and 14 Feb 2014. We used 'searchTwitter' function of the 'twitterR' package to search and download the tweets, based on the relevant hashtags and the accounts of many Australian EROs involved in the response to the event. In addition, information about individual users as well as communities and government emergency users' accounts relevant to the fire event were also captured.

Our preliminary investigation into Twitter posts (i.e., tweets) identified a number of terms and hashtags related to the fires. It included 'victoria bushfire', 'bush fire \#vicfire', '\#firevic' '\#firewatch' and '\#southmelbourne'. We used 'victoria bushfire', '\#Vicfire' and '\#firevic' to extract tweets specific to the fire and used by both the public and fire authorities to provide and exchange information. Accounts belonged to Australian emergency authorities (EROs) were considered formal and valid representatives of state and national level government. Therefore, tweets posted by these organizations were considered valuable having impact on the population. As outlined in the following section, a social network analysis was then conducted on the data to understand the activeness of the EROs and their influence on the followers during the event.

\footnotetext{
${ }^{1}$ http://www.sbs.com.au/news/article/2014/02/09/no-let-victorias-fire-emergency

${ }^{2}$ http://cran.r-project.org/web/packages/twitteR/index.html
} 


\section{RESULTS}

\subsection{Demographics findings}

Table 1 shows the list of top EROs involved in the 2014 Victorian bushfire. These EROs have joined Twitter as early as 2008, while some others have joined later in 2011 and 2012. The results indicate that all EROs have actively been sharing fire related information (updates, warnings and briefs) with the communities. As evident in Table 1, all ERO accounts have posted a large number of tweets since the creation of their accounts, with@Firewatch_Melb and @ Australianwarni posted the highest number of tweets since their establishment.

Table 1 also indicates that the information provided by the EROs through tweets is trusted by the individuals, as EROs' Twitter accounts have thousands of followers. There is a considerable variation among these accounts on followers and favourites. For example, @Firewatch_Melb and @ Australianwarni accounts, which posted the highest number of tweets every month (on average), have 1,014 and 1,704 followers respectively but the number of tweets that were declared favorites have been only 15 and 2 respectively. In comparison, @CFA_Updates and @MFB_News accounts have attracted a much higher number of followers (51 K and $12.2 \mathrm{~K}$ respectively), and 550 and 833 tweets respectively have been considered favorite by the people since the activation of these accounts. Surprisingly, these results indicate that older Twitter accounts have fewer posts every month yet with more favorites compared to the newer accounts, which have a higher number of postings every month (on average) but with a lesser number of favorites.

Table 1. Twitter profile of ERO accounts in Australia by Jan 2015

\begin{tabular}{|l|l|l|l|l|l|l|}
\hline \multicolumn{1}{|c|}{ User name } & \multicolumn{1}{|c|}{ Twitter join date } & $\begin{array}{c}\text { Total \# of } \\
\text { tweets }\end{array}$ & Following & Followers & Favourites & $\begin{array}{c}\text { Average } \\
\text { Tweets/pm } \\
\text { by Jan'15 }\end{array}$ \\
\hline @Firewatch_Melb & Aug 2012 & $78.3 \mathrm{~K}$ & 59 & $1 \mathrm{~K}$ & 15 & 2,610 \\
\hline @ Australianwarni & Nov 2011 & $60.5 \mathrm{~K}$ & 163 & $1.7 \mathrm{~K}$ & 2 & 1,551 \\
\hline @CFSAlerts & Dec 2010 & $40.8 \mathrm{~K}$ & 30 & $20.3 \mathrm{~K}$ & 20 & 816 \\
\hline @CFSTalk & Nov 2011 & $3.9 \mathrm{~K}$ & 9 & $2.5 \mathrm{~K}$ & 3 & 100 \\
\hline @Firewatch_VIC24 & Oct 2011 & $3.7 \mathrm{~K}$ & 49 & 144 & 5 & 92 \\
\hline @CFA_Updates & Nov 2008 & $40 \mathrm{~K}$ & 1082 & $51 \mathrm{~K}$ & 550 & 533 \\
\hline @Incident_Alert & April 2009 & $17 \mathrm{~K}$ & 69 & $6 \mathrm{~K}$ & 87 & 293 \\
\hline @MFB_News & Mar 2009 & $2.5 \mathrm{~K}$ & 731 & $12.2 \mathrm{~K}$ & 833 & 35 \\
\hline
\end{tabular}

Analysis of the activities of EROs and individual Twitter account holders during the 2014 Victoria fire revealed that altogether 2,685 Twitter accounts (users) were involved in disseminating the information by posting 7,981 tweets during the fire event between the $20^{\text {th }}$ of January to the $14^{\text {th }}$ of February in 2014 . This means, on average, each account holder posted approximately three tweets in relation to the event. Further analysis into the frequency of the tweets, as shown in Table 2, revealed that 1,629 users (20\% of all users) had posted single tweet, and only 16 users (accounts) posted 40 tweets or more about this event.

\subsection{Geo-location information}

Geo-location information referring to street addresses, city names and country names of the tweets, was the next valuable information that was retrieved and analysed in this study. Geo-location information generally helps EROs and general public to assess their own situation as well as the overall situation in the area during a natural hazard (Poblet et al. 2017; Crooks et al. 2013). In addition, it helps authorities and emergency managers to assess validity of information posted and also to create virtual communities in order to provide more targeted information in the wake of such natural hazards (Vieweg et al. 2010). Geo-location can be identified either if the user have directly made it available with the tweets or can be deduced from IP addresses using any of the IP geolocation solution (Poese et al. 2011; Eriksson et al. 2010).

In this study, we focused on geo-location information that was provided either directly by the user or through the client application. The analysis of the tweets in this study revealed the geo-location information of only 846 tweets, which is nearly $11 \%$ of the total number of tweets. Out of 846 tweets with available geo-location information attached, 52 were from outside Australia. Other tweets were originated within the $40 \mathrm{KM}$ radius of the Melbourne City. It is worth mentioning that Firewatch Incident Feeds was a source of 737 tweets out of 846 
(87.1\%) geo-location information enabled tweets. Twitter for iPhone and Android were the source of $52(6.1 \%)$ and $30(3.1 \%)$ tweets respectively which was followed by Instagram that sourced $19(2.2 \%)$ tweets. The rest of the five sources posted 2 tweets at maximum in relation to this fire hazard. Thus, we did not find a great percentage of geo-location enabled tweets in this case study. Past studies i.e. Krishnamurthy et al. (2008) and Crooks et al. (2013), have also found that providing geo-location information is not a common practice in the areas where latest mobile technologies are easily and rapidly adopted.

\subsection{Institutional Vs. Non-Institutional Contributions}

Content analysis of tweets collected in this study revealed that 3,579 tweets of the EROs were re-tweeted by 444 users between the $7^{\text {th }}$ to $9^{\text {th }}$ of February 2014 . These re-tweets were about $45 \%$ of the total number of tweets, indicating a high level of influence of the institutional EROs on their followers. This is an overwhelming result indicating the enthusiasm of the Twitter users in spreading information coming from the EROs. For example, 53 tweets from @CFA_Updates (which refers to the official page of Victoria fire authority) were re-tweeted 501 times. The above finding also shows that about $55 \%$ of all tweets were from accounts other than EROs.

Figure 1 illustrates a social network of the re-tweeting behaviour of the users. The nodes of the graph are users and a link between each pair of nodes exists if any one of the users has re-tweeted the other user's tweet. The graph shows reputable sources of tweets and further re-tweeting of the tweets from such as CFA_Updates, ABCemergency, CFAAlerts and Australianwarni during the Victoria fire event. A closer look into the dense areas of the graph revealed that the majority of the tweets from CFA-Updates and Australianwarni went on more than six hops in re-tweeting; however tweets from other sources could not go beyond four hops in re-tweeting the information from sources such as CFAAlert and ABCemergency. The propagation graph also shows cycles at various places indicating that the information was commented, replied and passed on to others.

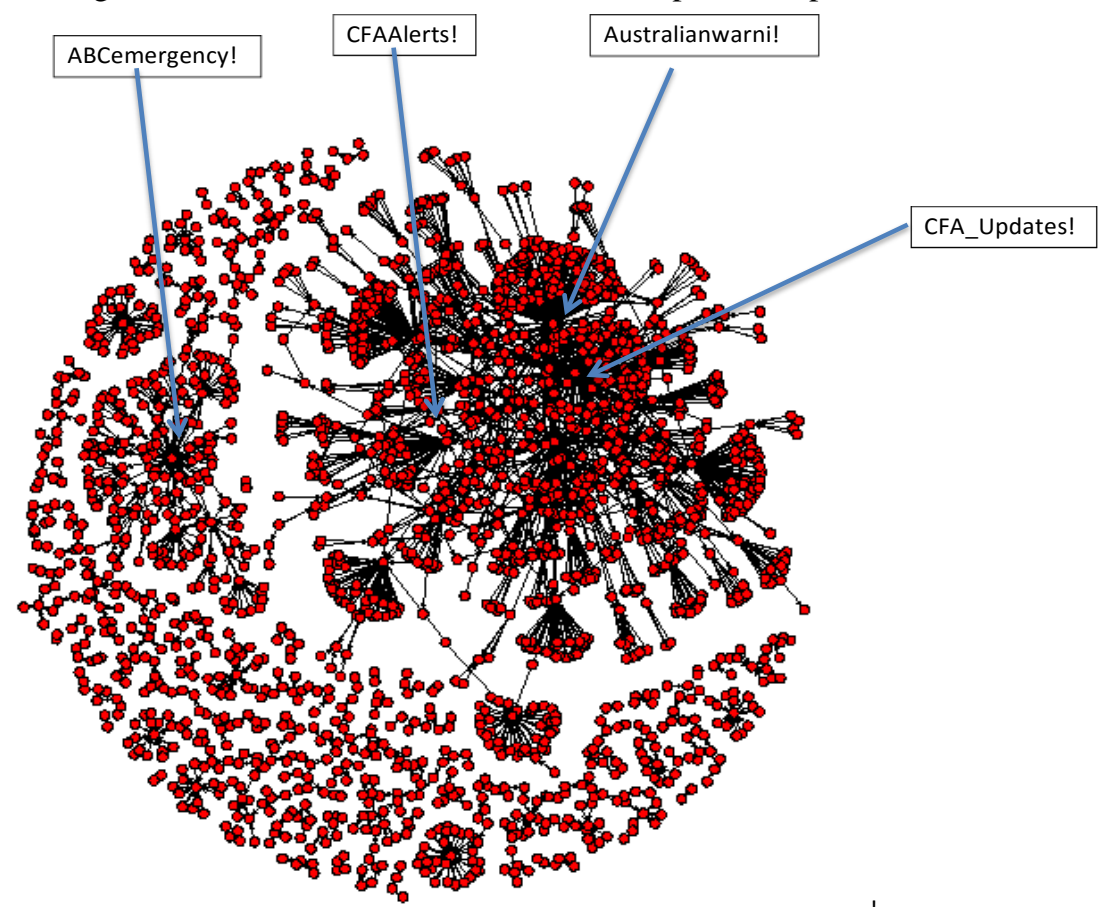

Figure 1: Re-tweet tree of Victoria bushfire 2014

Table 2 distinguishes institutional and non-institutional Twitter activities for the top 16 Twitter accounts. As this Table shows, about $75 \%$ of total tweets have been posted by institutional EROs; however there is a considerable variation among the number of tweeting, retweeting and mentioning of these accounts in their tweets. For example, @Firewatch_Melb and @ Australianwarni were the most active ERO accounts during the event, which posted 701 and 260 tweets respectively. On the other side, @MFB_News and @NSWRFS were the least active accounts, which posted 50 and 41 tweets during the event. This variation can be explained by the fact that since the fire occurred in the outskirts of Melbourne, @Firewatch_Melb and @ Australianwarni were the most responsible Twitter accounts in spreading fire related information. The @NSWRFS generally covers incidents from NSW, Victoria's neighbour state, and therefore they have been shown less active in relation to the Victoria fire.

Figure 2 also compares the number of 'Mentions' for institutional versus non-institutional accounts. As a common function in micrblogging services such as Twitter, Mention enables interactive postings by drawing attentions of other users (Tang et al. 2015). By mentioning users in a tweet, they receive notifications and possible 
retweeting by them may initiate a large cascade diffusion of the tweet. Mention can help users to improve the visibility of their tweets and expand their horizon of social interaction beyond their immediate reach (Wang et al. 2013). As an important interactive behaviour, Mention allows to actively direct a tweet to target users using the form of @username (Simon et al. 2014; Wang et al. 2013). Mentioning others in the tweets can also be viewed as an efficient way to pull (i.e. to initiate and encourage) interactions instead of push (i.e. visible only if you follow with passive responses). Therefore, instead of waiting passively for retweets after postings, Mention can actively initiate interactions at the time of the postings (Tang et al. 2015; Duan et al. 2010). Amongst all, three accounts had the highest number of Mentions: @CFSAlerts, @CFSAlerts, and @ geehall, two of which are EROs and one is a digital volunteer. However, as Figure 2 shows, the total number of institutional Mentions is significantly higher than those of non-institutional ones. This finding suggests that (i) people affected by this disaster have been and aware of EROs' Twitter account names and have actually used the information posted by these organizations, and (ii) they trusted the information posted by EROs since they mentioned EROs account names in their following tweets or re-tweets.

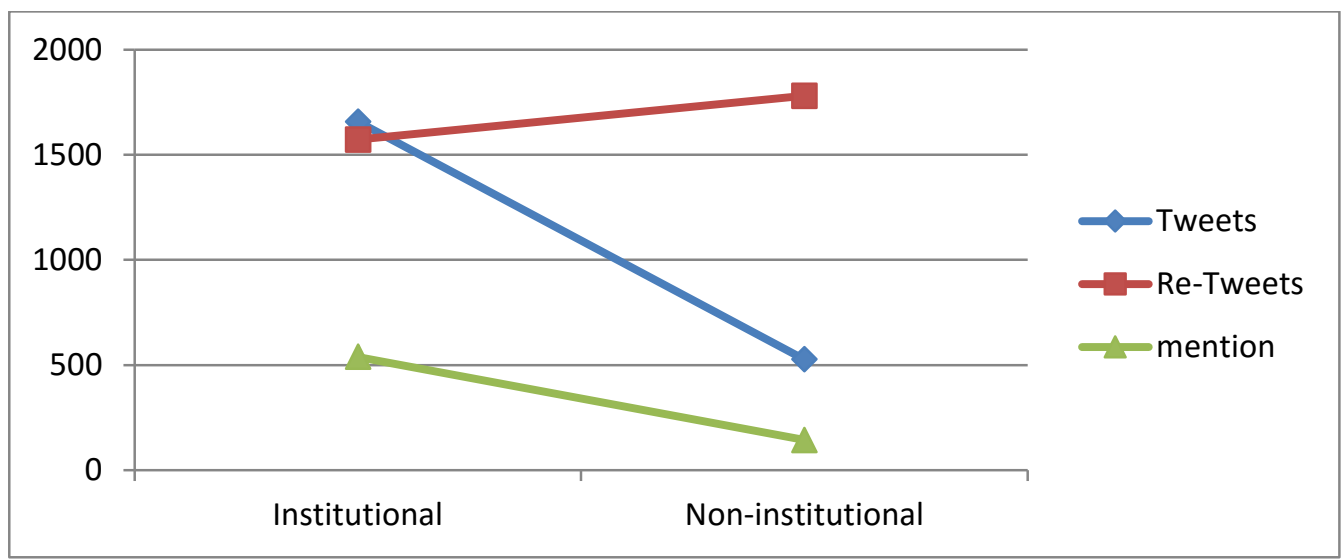

Figure 2: institutional vs. non-institutional information dissemination contributions

On the other hand, non-institutional actors have also played a significant role in information dissemination, with about $25 \%$ of all tweets were posted by them. As Figure 2 shows, while the total of number of tweets posted by non-institutional is less than EROs, the level of their re-tweets is significantly higher. This has important implications, as it reflects the community members' strong willingness to trust and share active individual Twitter account holders/social influencers' messages during a natural disaster. This is consistent with findings of other studies, as for example Cha et al. (2010) found that a high percentage of re-tweeting of tweets is an indicator of the level of influence of the users (who posted the messages) on their followers. In other words, the followers consider such a user a trusted source and regard the information posted by them valuable, notable and informative (Hughes and Palen 2009)

Table 2: Tweeting, Re-tweeting and Mentioning of Twitter accounts with over 40 posts

\begin{tabular}{|c|c|c|c|c|c|}
\hline Communities & User name & Description & $\begin{array}{l}\text { Total } \\
\text { \# of } \\
\text { tweets }\end{array}$ & $\begin{array}{l}\%(n) \text { of } \\
\text { RTs }\end{array}$ & $\begin{array}{l}\text { (n) of } \\
\text { mentions }\end{array}$ \\
\hline \multirow{11}{*}{$\begin{array}{l}\text { Institutional } \\
\text { news } \\
\text { distributors } \\
\text { (EROs) }\end{array}$} & @Firewatch_Melb & Local Fire authority & 701 & $0 \%(0)$ & 6 \\
\hline & @ Australianwarni & Fire authority & 260 & $15 \%(40)$ & 40 \\
\hline & @incident_alert & Local fire alert & 170 & $197 \%(335)$ & 156 \\
\hline & @CFSTalk & SA country fire services & 97 & $30 \%(30)$ & 57 \\
\hline & $@$ CFSAlerts & SA country fire services & 88 & $165 \%(146)$ & 152 \\
\hline & @ firewatch_VIC24 & Local Fire alert authority & 54 & $16 \%(9)$ & 3 \\
\hline & @ greatozgovtweet & Aus govt SM messages & 54 & $\begin{array}{l}1131 \% \\
(611)\end{array}$ & 4 \\
\hline & $@$ IncidentAlert16 & Fire authority & 51 & $0 \%(0)$ & 25 \\
\hline & @MFB_News & Fire authority & 50 & $42 \%(21)$ & 36 \\
\hline & @NSWRFS & NSW Govt fire authority & 41 & $39 \%(16)$ & 37 \\
\hline & @ ABCemergency & Media outlet & 87 & $414 \%(361)$ & 22 \\
\hline \multirow{2}{*}{$\begin{array}{l}\text { Non- } \\
\text { Institutional }\end{array}$} & @ geehall1 & Social media volunteer & 240 & $211 \%(507)$ & 135 \\
\hline & @ firey 81 & CFA volunteer & 154 & $421 \%(649)$ & 6 \\
\hline
\end{tabular}




\begin{tabular}{|c|c|c|c|c|c|}
\hline \multirow[t]{2}{*}{$\begin{array}{l}\text { news } \\
\text { distributors } \\
\text { (digital } \\
\text { Volunteers) }\end{array}$} & @VicStormChasers & $\begin{array}{l}\text { Severe weather specialist for } \\
\text { Victorians }\end{array}$ & 96 & $494 \%(475)$ & 0 \\
\hline & @neighbourday & Community representative & 36 & $416 \%(150)$ & 2 \\
\hline
\end{tabular}

Table 2 also shows a considerable variation among institutions' number of tweets and their re-tweets. While some EROs' tweets have achieved a higher level of re-tweets, others have performed the quiet opposite. For the example, tweets from @Firewatch_Melb were never retweeted by the other accounts. The tweets from @ Australianwarni were retweeted only 40 times in relation to this fire, which is a small percentage (15\%) of the total number of tweets posted by that account. Interestingly, 170 tweets from @incident_alert, were retweeted 335 times (197\% of the total number of tweets). The @ geatozgovtweet was one of the least active accounts (in terms of the number of posts) during the event, yet 54 of their tweets were retweeted 611 times, achieving the highest percentage $(1131 \%)$ in retweeting. Similar observations have been made for the other less active accounts. For example, 88 tweets from CFSAlerts were retweeted 146 times achieving a high percentage in retweeting frequency (165\%). The least active accounts such as @MFB_News and @NSWRFS have also shown a relatively high frequency rate $(42 \%$ and $39 \%$ respectively) for retweeting.

Furthermore, we found that the content of the tweets as well as the hashtags that EROs used played an important role in the total number of re-tweets. This means that tweets posted by government emergency organisations who covered the event from various perspectives and posted relevant contents were very attractive to community members. Also tweets from more appropriate hashtages have been retweeted more than the others. Similar observation has been made for the mentioning of the accounts. Similar to the pattern of re-tweeting, mentioning of very active accounts was less for the less active accounts as shown in Table 2. For example, @ firewatch_Melb and @ Australianwarni accounts that had tweeted 701 and 260 times were mentioned 85\% and $15 \%$ respectively. Some of the medium level active accounts such as @incident_alert, @CFStalks and @CFSalerts have been mentioned at a high rate of $91 \%, 58 \%$ and $172 \%$ respectively against their total number of tweets. Less active accounts such as@incident_alert16, @MFB_News and @NSWRFS have been mentioned at considerable rates, $49 \%, 72 \%$ and $90 \%$ respectively in the tweets from other accounts. The variation in tweeting, re-tweeting and mentioning of ERO accounts can be due to various reasons. For example, since the fire occurred in the outskirts of Melbourne, @ firewatch_Melb disseminated information more than the other ERO accounts. However, this does not mean that this account achieved a high number of retweeting and mentioning in the tweets from other people. Joining Twitter early in 2008 and 2009 and building trust among the communities can be among the reasons for some ERO accounts such as @CFA_updates, @incident_alert and @MFB_News to have their posts retweeted and mentioned more frequently than others.

In addition to some EROs, social media influencers, volunteers, and general public supporting communities also remained active on Twitter throughout the event. Our results revealed that these individual Twitter account holders have played a crucial role in the development of informed communities. For example, @ geehall1 (a volunteer) tweeted that "Murray Valley Hwy Ebden (11) and Huon (2 vehicles), NOT Yet Under Control", which was later twitter by @ABCemergency, a TV media outlet, about the situation in the following way "Halls Gap \#VIC residents advised to leave their homes ahead of wind change today". Our findings indicate that re-tweeting and mentioning of their postings have been highly frequent by the concerned communities because of the coverage of the event at a wider level and using appropriate hashtags related to the event.

\subsection{Tweet contents: informative rather than directive}

Altogether 1,566 tweets were posted by the selected 16 accounts in Table 2 . The majority of the tweets, particularly from EROs, explain the situation and what is happening on the ground. For example, @CFA_updates tweeted stating that “@theheraldsun: \#VICFires: It's not over yet. \#Melbourne wakes to smell of smoke as fires continue". Another tweet from @CFA-Updates informed the communities as "North Warrandyte fires now marked as under control - great work by local crews. Warning will be updated shortly \#vicfires".

However, such tweets were informative rather than directive. They did not provide directions in terms of what needs to be done in a particular situation for the safety of individuals and community members. Analysis of tweets revealed that the general public had shown their concern over the provision of limited information by the EROs. For example, a tweet from a citizen states that "It's scary when a cop knocks on your door \&amp; says "Fires are bad. You better pack your stuff \&amp; leave". Another citizen tweeted stating that "No luck escaping the smoke from \#vicfires Still horrible here. \#whereamiworking Phillip Island." 
A relatively high number of Mentions in Table 2 suggest that there is a considerable potential for the public to receive more directive information and guidance. Particularly, EROs have emphasised on the use of appropriate \#tags for streamlining the spreading of the fire related information. For example, one of the tweets posted by CFA_Updates is “@rexster: To all Vic tweeters, the \#tag being used by @CFA_Updates and others is \#firevic not \#vicfires pls RT and use for bushfire tweet". In this tweet, they appear to be instructing the Victorian public to retweet their postings with hashtag \#firevic not \#vicfires. Our findings show that determining an appropriate hashtag for the fire incident was challenging for the public. Analysis of the data also reveals that numerous hashtags were used in communicating this fire event, which can be categorised into three groups: the general level, state level, and local level. The most used general level hashtags to represent this fire event were \#bushfire, \#firewatch and \#bush. However, the state level hashtags included \#vicfires, \#firevic and \#victoria. These hashtags (general and state levels) existed and used on twitter prior to this event. People used hashtags such as \#southmelbourne, \#campbelfield, \#fishermansbend, \#epping, \#Oakpark and many others referring to the status of the fire at the local level. Thus, the communities used a variety of hashtags on twitter in relation to this fire. However, fire authorities preferred using \#firevic and requested people to use this hashtag in their postings in relation to the fire event.

\section{DISCUSSION}

\subsection{Disaster Information Dissemination Power Share}

Findings of this study revealed that while several Australian EROs have well-established twitter accounts and actively use them during disasters, a large portion of news dissemination activities on social media happens through non-institutional outlets and individual twitter account holders. This challenges traditional assumptions of the theories like World System Theory that assume information flows from large news outlets to smaller units. As shown in Figure 3, we propose a power share between institutional and non-institutional social media outlets during disaster events, which in turn calls for a more effective coordination between institutional (i.e. large organizations) and non-institutional (i.e. digital volunteers). Re-tweeting $45 \%$ of the tweets by EROs indicate that EROs had strong influence on their followers during the event and that the followers listened the instructions by the trustworthy EROs and re-tweeted the information, which went on many hops creating a social network analysis, as shown in Table 2. However, the power of non-institutional and digital volunteers becomes more apparent when the number of re-tweets for non-institutional and institutional accounts are compared for the 2014 Victorian bush fire. As shown in Figure 2, total of number of tweets by EROs are clearly more than the tweets from noninstitutional account holders, whereas they are quite the opposite in re-tweeting.

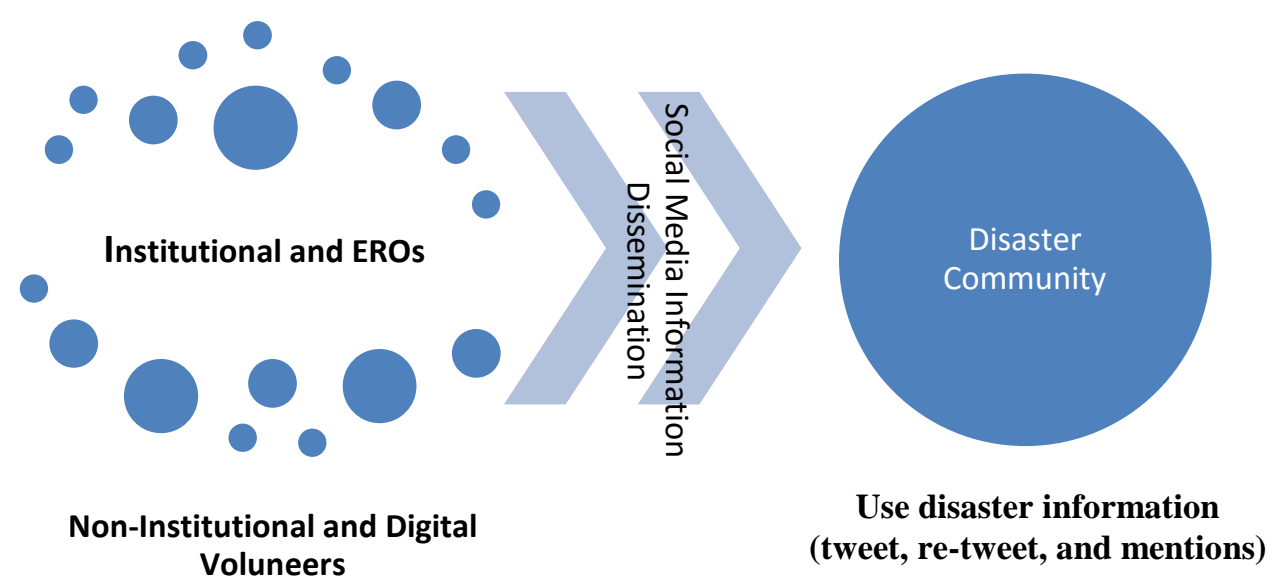

Figure 3: Disaster information dissemination power share

On the one hand, the results of this study confirm that that the role of EROs is critical in leading the communication among various stakeholders during natural disasters. While social media typically has the organic, groundswell stereotype as it relates to content generation and dissemination (Crooks et al. 2013; Vieweg et al. 2010), we found that formal institutions and hierarchies appear to maintain a sense of credibility and trust when social media users are searching for information concerning their personal safety during disasters. As Table 1 indicates, Australian EROs have overwhelmingly realised the importance of establishing Twitter accounts, and that the newer ERO accounts are more active than the older ones in posting messages during natural hazards. The results provided in Table 1 also suggest that the older accounts have a greater number of followers and favourites 
for their postings, which may mean an early embark on twitter has helped some EROs to win the trust of the communities over newer EROs. With more than 2.8 million active users (to date) in Australia, presence on Twitter platform has essentially become a need for EROs to communicate with the communities during natural disasters/hazards (Sinnappan et al. 2010).

On the other hand, the findings of this study also revealed that almost $50 \%$ of all messages exchanged on Twitter about 2014 Victorian bush fire were initiated by non-EROs and non-institutional account holders. This is consistent with some other studies in the literature suggesting that the general public is the main player in communicating the events related information on social media (Vieweg et al. 2010; Oh et al. 2013; Simon et al. 2014). As table 2 presents, accounts such as@geehall1 and @ neighbourday are volunteers, actively posted information about this event, and also had their posts re-twitted on a very large scale. Digital volunteering is a recognized phenomenon in the context of social media. Starbird et al. (2011) considers digital volunteers individuals or groups who predominantly monitor incoming digital feeds to find new, relevant, and actionable information (Zao et al. 2011). Cobb et al. (2014) have recognized the importance of processing real-time and firsthand information from the members of a disaster-affected-community for a better decision making and designing strategies. Digital volunteers often work to treat the social media data into useable resources. Cobbs et al. (2014) conducted contextual interview with digital volunteers and emergency managers to provide insights into their collaboration and the use of tools for monitoring disasters. The results of their study highlight the importance of digital volunteers' impact on design implications of support systems that are used in coordinating and integrating disaster management activities.

\subsection{Institutional/non-institutional coordination for information dissemination}

Further analysis of the tweet data indicated that there was peak and off peak time of the tweets by EROs. There were hours of the day when more than 150 tweets were posted whereas in other hours only a couple tweets were posted about the event. This great variation in the frequency of tweets indicates that EROs may not have allocated sufficient resources to conduct consistent communication around the clock. Extremely low tweeting rates in particular hours, or in other words less activeness from EROs in particular hours could have created opportunities for people to spread suspicious information or rumours (Oh et al. 2013). Therefore, EROs need to take an active role in consistent communication with the disaster affected community as well as with active digital volunteers that post and share disaster information on social media. This specially becomes important, as a relatively higher number of Mentions suggest that people trust the information posted by EROs, take appropriate actions accordingly, and forward the corresponding messages to other affected people in the community.

Furthermore, the contents of most postings were informative rather directive for the affected communities. This means tweet posts were mainly about updating the communities about the fire situation on the ground, and less about directing the community members with what actions they need to perform for their safety. Safety of the communities is the mission of Australian EROs and how social media can be used to support the safety of the communities is still a challenging task for the EROs (Crooks et al. 2013). Our findings suggest that pointing to useful resources under a particular event condition can be helpful for the communities to enhance their safety. To point to the right resource promptly, EROs might have to establish communication links with other support agencies such as Red Cross and Salvation Army during the disasters as well as active digital volunteers/Twitter influencers.

The above finding becomes especially important as the information posted by the non-institutional and individual Twitter accounts may sometimes be unreliable, leading to rumours among the communities (Popoola 2013). Rumours and suspicious information can be neutralised if EROs play an active role on social media during extreme events. It is evident from the results of this study that the active role played by the EROs during the Victorian fire event had helped to build public trust on EROs. The information that came from EROs on the fire event was spread by the public based on instructions provided by the EROs. Thus, development of a closer and more trustful communication system between the EROs and active Twitter influencers can significantly help to reduce rumours about the event.

\section{CONCLUSION AND RECOMMENDATION FOR FUTURE STUDIES}

To the best of our knowledge, this is the first study that has distinguished the use of Twitter by formal institutions (e.g. Australian EROs) from non-institutional (e.g. digital volunteers) during a natural disaster. This study primarily analysed the services provided by major Australian EROs as well as active individual account holders through a social media platform, Twitter, during a fire event in 2014.

The results of this study showed EROs' strong and active presence at social media, particularly at Twitter. This has helped them to play a leading role in communicating with their followers on the fire event, which has 
been evidenced by a high level of posted tweets. In comparison, non-institutional account holders have often posted a larger number of shares and re-tweets than EROs. This in turn reveals the high influence that noninstitutional channels play in disaster information dissemination during a natural event. Thus, future research is needed to further examine frameworks or mechanism for a more effective coordination or collaboration between institutional versus non-institutional social media accounts for dissemination of disaster information. Moreover, future studies should investigate why non-institutional messages may get more viral than some EROs. Research is needed into understanding factors that may influence share-ability of institutional vs non-institutional warnings during a disaster.

Another important result of this study was that the ERO's tweets were primarily used to inform affected people about the status of the disaster rather directing them to take particular actions, even when such tweets came from institutions invested with the power to direct. This could be because EORs may consider Twitter as a tool for information dissemination than a channel for giving specific directions to the people who may need different advice in different circumstances. However, past studies in the use of social media in natural disasters have emphasized on the potentials of Twitter and social media for supporting official communications and reflecting ground level conditions (Sinnappan et al. 2010). Thus, future research is needed to investigate on EROs' perception and intention of using social media channels, and Twitter in particular, during disasters, and what protocols they may or may not have for using these tools for the purpose of providing directions and official advice.

Furthermore, while social media platforms work very well in broadcasting trustworthy information (Erfani et al., 2017), they are also effective in spreading baseless rumours which can contribute to the chaos in the affected areas (Mendoza et al. 2010). Yates and Paquette (2011) have questioned the credibility of some social media feeds during natural disasters. They argue that during a natural disaster and when information from official sources is scarce, rumours can be spread on social media contributing to the chaos on the ground. Popoola (2013) discussed that users generally have difficulty discerning truthfulness of tweets based on their contents. To develop a deeper understanding of the difference between valid news and rumours, Mendoza et al (2010) collected 4,727,524 tweets on Chilean earthquake from February 27 to March 2 in 2010 and conducted two studies to investigate (i) how the news and rumours propagate through networks, and (ii) the ability of the network to discriminate between false rumours and confirmed news. Their results postulated that the propagation of tweets that correspond to rumours differs from tweets that spread news in a way that false rumours are questioned much more than the confirmed truths by the users (also see Popoola (2013)). From this perspective, it appears that noninstitutional and less formal Twitter account holders may act as a collaborative filter of information that does not allow rumours to penetrate deep in the social graph. Future research is therefore needed to deepen our understanding on how to distinguish rumours from trustworthy news during a disaster, and that how institutional and non-institutional people can work together to avoid or control spreading false information into the community.

More research is also needed to study the integration of general public's tweets into institutional applications, which in turn may drive innovation in services and communication with the public. Rosenberger et al. (2016) investigated the use of social media platforms such as Twitter by institutional organizations, and found that linking such platforms with their internal business applications can lead to various benefits, including transparency in actions, improving citizens' public participation, and improvement in services.

Lastly, our findings showed that while most of EROs and active digital volunteers posted geo-enabled tweets, only a small percentage of general public shared geo-enabled tweets. For example, Firewatch Incident Feed alone was the source of $87.1 \%$ of the geo-enabled tweets. With respect to the general public, the results are consistent with the results of past studies on geo-location such as Crooks et al. (2013) and Krishnamurthy et al. (2008) suggest that general public does not consider posting geo-enabled tweets during extreme events. Geolocation information referring to street addresses, city names and country names of the tweets is a valuable information for EROs and general public to assess their own situation as well as the overall situation in the area during a natural disaster/hazard (Crooks et al. 2013). In addition, it helps EROs to create virtual communities in order to provide more targeted information in the wake of such events (Vieweg et al. 2010). Further research is thus needed to investigate whether and how users can be motivated for using using geo-location enabled tweets during a disaster, and in what ways geo-location enabled tweets may further assist EROs in supporting the disaster affected community.

\section{REFERENCES}

Abedin, B., Babar, A., \& Abbasi, A. (2014). Characterization of the Use of Social Media in Natural Disasters: A Systematic Review. In Big Data and Cloud Computing (BdCloud), 2014 IEEE Fourth International Conference on, (pp. 449-454).

Abedin, B. Jafarzadeh H. (2013). Attracting and retaining customers on Facebook business pages: a content analysis of an online discussion forum. International Journal of Technology Marketing, 8(3), 304-315. 
Abedin, B. (2016). Diffusion of adoption of Facebook for customer relationship management in Australia: An exploratory study. Journal of Organizational and End User Computing (JOEUC), 28(1), 56-72.

Queensland police service-media and public affair branch (2011). Disaster management and social media - a case study, version 1.0.

Ahmad, A. (2011) Use of social media in disaster management. Second international conference on information systems (ICIS), Paper 16.

Alam, S. L. \& Walker, D. (2011). The public Facebook: A case of Australian Government Facebook pages and participation. ACIS, 2011, Sydney.

Barnett, G. A. (2001). A longitudinal analysis of the international telecommunication network. American Behavioral Scientist, 44(10), 1638-1655.

Bird, D., Ling, M., \& Haynes, K. (2012). Flooding Facebook-the use of social media during the Queensland and Victorian floods. Australian Journal of Emergency Management, 27(1), 27.

Butts, C. T. 2008. Social network analysis with sna. Journal of Statistical Software, 24(6), 1-51.

Cha, M., Haddidi, H., Benevenuto, F. \& Gummadi, K. P. (2010) Measuring user influence in Twitter: The million follower fallancy. 4th International AAAI Conference on Weblogs and Social Media ICWSM, 2010 Washington, DC.

Chongsuvivatwong, V. (2008). Analysis of epidemiological data using R and epicalc. Thailand, Epidemiology Unit Prince of Songkla University.

Cobb, C., McCarthy, T., Perkins, A., Bharadwaj, A., Comis, J., Do, B., \& Starbird, K. (2014). Designing for the deluge: understanding \& supporting the distributed, collaborative work of crisis volunteers. In Proceedings of the 17th ACM conference on Computer supported cooperative work \& social computing, 888-899.

Crooks, A., Croitoru, A. \& Stefanidis, A. (2013). \#earthquake: Twitter as a distributed sensor system. Transactions in GIS, 17(1), 124-147.

Diaz-Uriarte, R. \& Andres, S. A. D. (2006). Gene selection and classification of microarray data using random forest. BMC Bioinformatics, 7, 3 .

Duffy, N. (2012). Using social media to build community disaster resilience. Australian journal of emergency management. 27(1), 40-45.

Ehnis, C. \& Bunker, D. Social media in disaster response: Queensland Police Service - public engagement during the 2011 floods. 23rd Australasian conference on information systems, 2012 Geelong, December 3-5.

Erfani, S. S., Blount, Y., \& Abedin, B. (2016). The influence of health-specific social network site use on the psychological well-being of cancer-affected people. Journal of the American Medical Informatics Association, 23(3), 467-476.

Erfani, S.S., Abedin, B. \& Blount, Y. (2017). The effect of social network site use on the psychological well-being of cancer patients, Journal of the Association for Information Science and Technology, 68(5), 1308-1322.

Eriksson, B., Barford, P., Sommers, J. \& Nowak, R. (2010). A learning-basedapproach for IP geolocation. Kishnamurthy A and Plattner B (eds) passive and active measurment, Berlin, Springer Lecture Notes in Computer Science 6032, 171-180.

Falls, A. D. (2013). Brand management in social media environments: an institutional theory perspective (Doctoral dissertation, University of Ulster).

Fothergill, A., Maestas, E. G. \& Darlington, J. D. (1999). Race, ethnicity and disasters in the United States: A review of the literature. Disasters, 23(2), 156-73.

Golan, G. J., \& Himelboim, I. (2016). Can World System Theory predict news flow on twitter? The case of government-sponsored broadcasting. Information, Communication \& Society, 19(8), 1150-1170.

Heyde, S. V. D., Sonntag, J., Kaschek, D., Bender, C., Bues, J., Wachter, A., Timmer, J., Korf, U. \& Beibarth, T. (2014). RPPanalyzer Toolbox: An improved R package for analysis of reverse phase protein array data. BioTechniques, 57, 125-135. 
Houston, J. B., Hawthorne, J., Perreault, M. F., Park, E. H., Goldstein Hode, M., Halliwell, M. R. \& Griffith, S. A. (2015). Social media and disasters: a functional framework for social media use in disaster planning, response, and research. Disasters, 39(1), 1-22.

Hu, M. \& Liu, B. (2004) Mining and summarizing customer reviews. Proceedings of the tenth ACM SIGKDD international conference on Knowledge discovery and data mining, 168-177.

Hughes, A. L. \& Palen, L. (2009) Twitter adoption and use in mass convergence and emergency events. Proceedings of 6th International ISCRAM Conference, Gothenburg Sweeden.

Kavanaugha, A. L., Foxa, E. A., Sheetzb, S. D., Yanga, S., Lid, L. T., Soemakerc, D. J., Natsevf, A. \& Xieg, L. (2011) Social media use by government: From the routine to the critical. The Proceedings of the 12th Annual Internaitonal Conference on Digital Government Research, June 12-15 College Park, MD, USA, 121-130.

Krishnamurthy, B., Gill, P. \& Arlitt, M. (2008) A few chirps about twitter. WOSN, Seattle, Washington, USA.

Lee, J., Agrawal, M., \& Rao, H. R. (2015). Message diffusion through social network service: The case of rumor and non-rumor related tweets during Boston bombing 2013. Information Systems Frontiers, 17(5), 9971005.

Mandel, B., Culotta, A. \& Boulahanis, J. (2012) A demographic analysis of online sentiment during Hurricane Irene. Proceedings of the 2012 Workshop on Language in Social Media, Montreal, Canada. 27-36.

May, R. L. (2009). Australian Twitter hits all time high ZDnet Retrieved 14 June 2010 from http://m.zdnet.com.au/australian-twitter-use-hits-all-time-high-339294382.htm

Mcnerthney, C. (2012). Shooting Suspect Visited Bar Before; Gave Off a ‘Bad Vibe. Seattle Pl.

Mendoza, M., Poblete, B. \& Castillo, C. (2010) Twitter under crisis: Can we trust what we RT? 1st workshop on social media, July 25, Washington DC.

Mills, A., Chen, R., Lee, J. \& Rao, H. R. (2009). Web 2.0 emergency applications: How useful can Twitter be for emergency Response. Journal of Information Privacy and Security, 5(3), 3-26.

Oh, O., Agrawal, M. \& Rao, H. R. (2013). Community Intelligence and social media services: A rumour theoretic analysis of tweets during social crises. MIS quarterly, 37(2), 407-426.

Pandey, N. \& Natarajan, S. (2016). How social media can contribute during disaster events? Case study of Chennai floods 2015. In Advances in Computing, Communications and Informatics (ICACCI), IEEE International Conference on, 1352-1356.

Poblet, M., García-Cuesta, E. \& Casanovas, P. (2017). Crowdsourcing roles, methods and tools for data-intensive disaster management. Information Systems Frontiers, 1-17.

Poese, I., Uhlig, S., Kaafar, A. M., Donnet, B. \& Gueys, B. (2011). IP geolocation database: Unreliable? computer communication review, 41(2), 53-56.

Popoola et al. (2013). Information verification during natural disasters. IW3C2, Rio De Janerio, Brazil

Rosenberger, M., Lehrer, C. \& Jung, R. (2016). Integrating data from user activities of social networks into public administrations. Information Systems Frontiers, 1-14.

SBS Australia. (2014). http://www.sbs.com.au/news/article/2014/02/09/no-let-victorias-fire-emergency

Scott, W. R. (1995). Institutions and Organizations. Thousand Oaks, CA: Sage.

Simon, T., Goldberg, A., Aharonson-Daniel, L., Leykin, D. \& Adini, B. (2014). Twitter in the cross fire - the use of social media in the Westgate Mall terror attack in Kenya. PLOS ONE, 9, 1-11.

Sinnappan, S., Farrell, C. \& Stewart, E. (2010b) Priceless Tweets! A Study on Twitter Messages Posted During Crisis: Black Saturday. ACIS, 1-10 Dec 2010b, Brisbane, Australia.

Starbird, K. \& Palen, L. Voluntweeters: Self organizing by digital volunteers in times of crisis. CHI, 2011. 10711080.

Starbird, K. \& Palen, L. (2013). Working \& sustaining the virtual disaster desk. Proceedings of CSCW, 491-502.

STieglitz, S. \& Kruger, N. (2011) Analysis of Sentiments in Corporate Twitter Communication - A case study on an issue of Toyota. Proceedings of 22nd Australian Conference on Information Systems, November 30December 22011 Sydney, Australia. 
Tang, L. Ni, Z., Xiong, H., \& Zhu, H. (2015) Locating targets through mention in Twitter. World Wide Web, (2015), 18, 1019-1049.

Vieweg, S., Hughes, A. L., Starbird, K. \& Palen, L. (2010). Microblogging during two natural hazards events: What twitter may contribute to the situational awareness. CHI 2010, Atlanta Georgia, USA.

Wallerstein, I. (1974). The modern world system. New York, Academic Press.

Wang, B., Wang, C., Bu, J., Chen, C., Zhang, W. V., Cai, D. \& He, X. (2013) Whom to Mention: Expand the Diffusion of Tweets by @ Recommendation on Micro-blogging Systems. International World Wide Web Conference, May 13-17, 2013, Rio De Janeiro.

Whitelaw, T. \& Henson, D. (2014). All that I'm hearing from you is white noise': social media aggregation in emergency response. Australian Journal of Emergency Management, 29, 48-51.

Yates, D. \& Paquette, S. (2011). Emergency knowledge management and social media technologies: A case study of the 2010 Haitian earthquake. International Journal of Information Management, 31(1), 6-13.

Yin, J., Lampert, A., Cameron, M., Robinson, B. \& Power, R. (2012). Using social media to enhance emergency situation awareness. IEEE Intelligent Systems, 27(6), 52-59.

Zhao, Y. \& Cen, Y. (2014). Data Mining Applications with R, Elsevier.

Zao, S. et al. (2011) Human as real-time sensors of social and physical events: A case study of Twitter and sport games. Houston, TX, Rice University Technical Report No. TR0620. 


\section{Authors' Biography}

Dr Babak Abedin holds a PhD in Information Systems from the University of New South Wales. His research focus is on how social information systems and online communities can empower individuals and transform organizations. He has widely published in this domain, most recently in Journal of the Association for Information Science and Technology, Journal of the American Medical Informatics Association, Interactive Learning Environment.

Dr Abdul Babar is an academic researcher at Sydney University, Sydney. He has extensive industry and research experience in business strategy analysis \& design and its translation into IS development encompassing telecom, retail and banking industry projects. With the strategic view of IS development, Dr Abdul Babar is currently focused on industry related research projects in the area of disaster management, social media and IS outsourcing 